\title{
GAINS AND LOSSES OF HLA CLASS II (DR) AND CD4 IN ATYPICAL HYPERPLASIA, CARCINOMA IN SITU AND INFILTRATING DUCTAL CARCINOMA OF THE BREAST
}

\author{
Demetrio Tamiolakis ${ }^{1}$, Ioannis Venizelos ${ }^{2}$, Maria Lambropoulou ${ }^{4}$, Theodoros Jivannakis ${ }^{4}$, Evagelia Seliniotaki ${ }^{1}$, \\ Panagiotis Tsikouras ${ }^{5}$, Vasilios Limberis ${ }^{5}$, Angelos Tsalkidis ${ }^{3}$, Nikolas Papadopoulos ${ }^{3}$
}

General Hospital of Chania: Department of Cytology1; Ippokration Hospital of Salonica: Department of Pathology ${ }^{2}$; Democritus University of Thrace: Department of Histology - Embryology ${ }^{3}$; Department of Obstetrics and Gynecology ${ }^{5}$; General Hospital of Drama: Department of Pathology ${ }^{4}$

Summary: Aim: Breast cancer is a frequent cause of death among women with gynaecologic malignancies despite the introduction of combination chemotherapy. There is therefore a need for new therapeutic strategies for patients with breast cancer, such as cellular immunotherapy. In this immunohistochemical study we analyzed the epithelial expression of major histocompatibility complex (MHC) class II (HLA-DR) on atypical and malignant primary mammary epithelial cells, as well as the magnitude of the stromal T lymphocytes (T4 subset) at the tumor site. Experimental design: The study was carried out retrospectively in tumor tissue from 82 patients with mammary lesions (31 cases of atypical ductal hyperplasia -ADH-, 12 cases of ductal carcinoma in situ -DCIS- and 39 cases of infiltrating ductal carcinoma not otherwise specified -IDC-NOS). Medullary carcinomas were not included in our investigation. Material used had been formalin fixed and paraffin embedded. Results: HLA class II (DR) was expressed in 20 of 31 ADHs (64.5\%), in 4 of 12 DCISs (33.3\%), and in 10 of 39 IDC-NOSs (25.6\%). CD4 was expressed in 9 of 31 ADHs (29\%), in 5 of 12 DCISs (42\%), and in 26 of 39 IDCNOSs (67\%). Conclusions: The results showed decreased epithelial expression of HLA class II (DR) and increased stromal expression of CD4, as the lesion progressed to malignancy. Gradual loss of epithelial HLA class II expression might be a manifestation of cellular differentiation from the atypical form versus the malignant one, signaling simultaneously a selective effect on the response capacity of the immune system.

Key words: HLA class II (DR); CD4; Atypical ductal hyperplasia of the breast; Breast cancer

\section{Introduction}

Studies of the relation between the tumour and host immune systems have shown that major histocompatibility class (MHC)-I antigen expression, normally present in all nucleated cells, is reduced in malignancies $(11,20,21,26$, 41). This feature was related to tumour progression in experimental tumour systems $(18,43,46)$. Furthermore, human leukocyte antigen (HLA)-DR, a class II MHC antigen, which is normally expressed only in antigen presenting cells of the immune system (24), shows variable expression in malignancies $(16,30)$.

The examination of MHC antigen expression in breast carcinoma in particular has been prompted by the observation that lymphocytic infiltrates occur in two thirds of these malignancies (22), suggesting that the immune system may be active in the modulation of tumour behavior. MHC-I expression in breast cancer was found to be hetero- geneous, but usually decreased $(29,47,48,49)$. In some studies prominent MHC-I expression was related to better tumour differentiation $(47,49)$, and a favorable prognosis (5), whereas in other no such correlation's were found $(6,31,37,44)$. Regarding HLA class II (DR) expression in breast cancer, most studies report intermediate values $(1,4,25,29,31,32,49,50)$, but the findings are variable $(3,22)$. HLA class II (DR) expression may also be related to tumour grade; two studies reported that none of the carcinomas that were negative or only focally positive were well differentiated $(4,50)$. However, several other studies failed to support this finding $(25,31,49)$. The link between HLA class II (DR) expression and prognosis also remains unclear $(29,37,48)$

Increasing evidence of a critical role for the $\mathrm{T}$ helper cell in initiating, regulating, and maintaining antitumour immune responses (40) justifies more investigation of HLA class II. HLA-DR (class II) expression by primary breast 
cancers is positively correlated with the differentiation state of the tumour and the expression of progesterone receptors (both associated with good prognosis). Furthermore, in medullary carcinoma of the breast (generally associated with a good prognosis and a florid $\mathrm{T}$ lymphocyte response) the HLA class II (DR) expression was $74.5 \%$ in primary tumours and $67.3 \%$ in nodal metastases, compared to $17.7 \%$ and $7 \%$ in ductal carcinomas (27). It is not hard to imagine how loss of HLA class I expression may aid escape from immune surveillance but HLA class II antigens (which are normally presented to $\mathrm{T}$ lymphocytes by professional antigen-presenting cells, not tumour cells) may not be subject to such easy down-regulation by the tumour itself and therefore specific MHC class II molecules may be more likely to confer variability in cancer susceptibility than MHC class I molecules (19).

The current study determined the HLA class II (DR) expression of atypical ductal hyperplasia, ductal carcinoma in situ and infiltrating ductal carcinoma of the breast with quantification of stromal infiltration by CD4+ T lymphocytes, with aim to allow future assessment of whether HLA class II expression has an impact on clinical outcome.

\section{Materials and methods}

Tumor samples were obtained from 82 patients ranging in age from 35-85 years. The mean (SD) age of our patients was 57.8 years, and mean tumor size in the cases of IDC-NOS was $2.4 \mathrm{~cm}$ (range 0.9 to $6.5 \mathrm{~cm}$ ). All our selected cases were recovered from routine histological files. 15 patients were premenopausal. The 82 tumours studied included 31 cases of atypical ductal hyperplasia (ADH), 12 cases of ductal carcinoma in situ (DCIS) micropapillary pattern, and 39 cases of infiltrating ductal carcinoma not otherwise specified (IDC-NOS) of the breast. Our material included cases with an abundant stromal lymphocytic infiltrate. The 31 cases of ADH and 12 cases of DCIS were an accidental finding in patients which had been operated for fibrocystic chances of the breast. Stage of disease at presentation (in the cases of IDC-NOS) was known in 23 patients; most (70\%) had stage II disease, and the remainder were divided equally between stage I and stage III. Fifteen patients had lymph node metastases. The specimens had been fixed in $10 \%$ formol-saline and processed routinely through absolute alcohol and xylene before embedding to paraffin wax. Conventional histological stains included hematoxylin and eosin. Regional Committees of Ethics approved the study. Written informed consent was obtained from all patients, and the procedures followed were in accordance with the institutional guidelines.

\section{Immunohistology}

Additional slides were stained with several monoclonal antibodies that are reactive in paraffin sections for immunohistochemical studies. An antigen-retrieval method using a pressure cooker was performed before immunohistochemical staining (34). The staining consisted of a first-stage incubation with the following primary monoclonal antibodies: HLA class II (DR) (TAL.1B5); CD20 (L26); CD4 (1F6); CD8 (C8-144). Bound antibodies were visualized employing the alkaline phosphatase anti-alkaline phosphatase (APAAP) method (9) and Fast Red for development. Negative controls were omission of primary antibody. Positive controls for anti-HLA II (DR) and anti-CD4 antibody were the staining of stromal cells and the tissue of tonsils respectively. We focus our attention on HLA class II (DR), and CD4 antibodies since the others antigens were beyond the scope of our study.

The immunostained sections were examined with an X 40 objective and the distribution of HLA class II (DR) and CD4 within the cell was recorded. To count the number of cells with HLA II (DR) and CD4 staining, a 10 X 10 square calibrated grid was inserted into the eyepiece of an Olympus BX40 binocular microscope.

Five-to-ten fields were examined for each section, and at least 1000 cells were scored, depending on cellularity. The percentage of positive cells was recorded as the HLA class II (DR) and CD4 indices.

$$
\begin{aligned}
\text { HLA-II }(\text { DR }) \text { index } & =\frac{\text { No of positive cells }}{\text { No total }(\text { positive+negative cells })} \\
\text { CD4 index } & =\frac{\text { No of positive cells }}{\text { No total }(\text { positive+negative cells })}
\end{aligned}
$$

The indices ranged from $0-100 \%$, with a mean of $18 \%$. The mean index was evaluated in three ranges: low index (under $18 \%$ ), grade I; moderate index (from 18 to $50 \%$ ), grade II; and high index (from 51 to $100 \%$ ), grade III.

For all cases both the percentage and intensity of HLA class II (DR) staining were numerically scored as in Table 1. Particular emphasis was given on the total percentage of epithelium stained, the intensity of staining and uniformity. Intensity was derived by comparison of epithelial staining with stromal cell reactivity.

Tab. 1: Scoring system for HLA class II (DR) expression.

\begin{tabular}{|l|c|}
\hline Percentage of cell expression & score \\
\hline 0 & 0 \\
18 & 1 \\
50 & 2 \\
100 & 3 \\
\hline Intensity of HLA class II (DR) expression & score \\
\hline Negative & 0 \\
Very weak, just detectable & 1 \\
Readily detectable & 2 \\
Just less intense than stromal cells & 3 \\
Strong intensity, equal to stromal cells & 4 \\
\hline
\end{tabular}




\section{Results}

The sections were examined independently by two observers, and positive cellular staining for HLA class II (DR) and CD4 antigens were manifested as fine red cytoplasmic expression.

HLA class II (DR) was expressed in 20 of 31 of atypical ductal hyperplasias (ADH) (64,5\%) (Fig. 1), in 4 of 12 ductal carcinomas in situ (DCIS) (33.3\%) (Fig. 2), and in 10 of 39 infiltrating ductal carcinomas not otherwise specified (IDC-NOS) (25,6\%) (Fig. 3). CD4 was expressed in 9 of 31 ADHs (29\%) (Fig. 4), in 5 of 12 DCISs (42\%), and in 26 of 39 IDC-NOS (67\%) (Fig. 5). There was a variable reduction of the intensity and proportion of epithelial staining from ADH towards IDC-NOS. On the contrary, there was a variable increase in the numbers of CD4 positive stromal infiltrates from ADH towards IDC-NOS. HLA class II (DR) expression by malignant epithelium showed the greatest change from that seen in $\mathrm{ADH}$. In 10 cases of

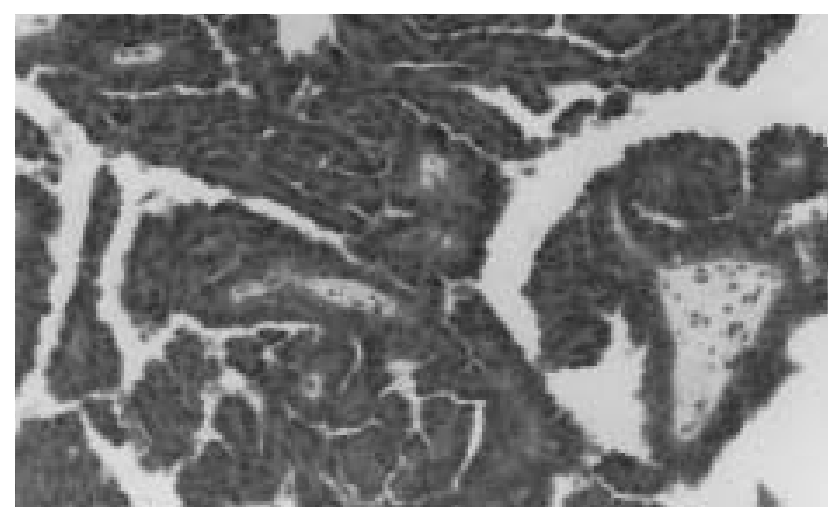

Fig. 1: HLA class II (DR) expression in atypical ductal hyperplasia $(\mathrm{ADH})$ with micropapillary pattern. Immunostaining using APAAP technique (red labeled cells). Original magnification X200.

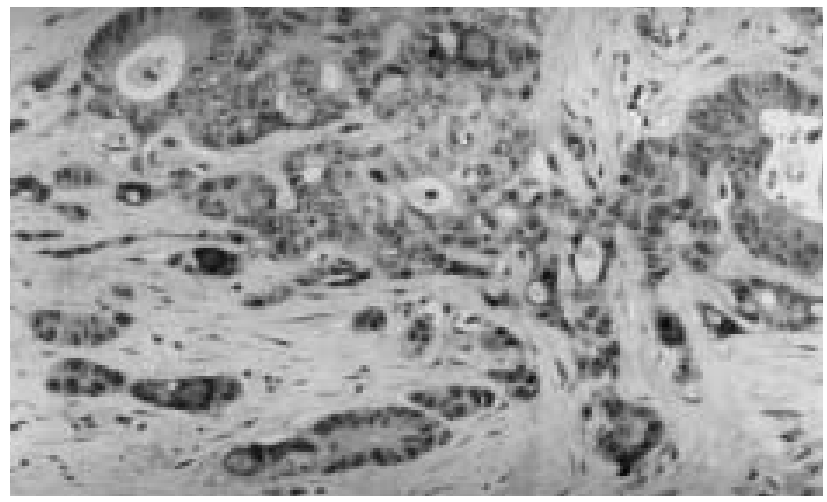

Fig. 3: HLA class II (DR) expression in infiltrating ductal carcinoma (IDC-NOS). Immunostaining using APAAP technique (red labeled cells). Original magnification X200.
IDC-NOS, 3 cases exhibited epithelial HLA class II (DR) expression in a manner similar to that seen in ADH, and 7 cases showed variable reduction of the intensity and numbers of epithelial cells stained. From the 4 cases of DCIS 2 cases showed HLA class II (DR) expression as in $\mathrm{ADH}$ and 2 exhibited variable expression.

Lymphocytes - Stromal cells identified with CD20, CD4, and CD8 were morphologically lymphocytes. Lymphocytes were predominantly in periductal and intralobular connective tissue in benign $\mathrm{ADH}$. Both $\mathrm{B}$ and $\mathrm{T}$ lymphocytes were identified in a ratio of approximately $1: 3.5$, and with slightly more CD4 than CD8 positive cells. Interepithelial cells were also present, the vast majority of which were T lymphocytes, mainly CD4. In DCIS and IDC-NOS, lymphocytes were predominantly in stroma around tumour cell islands, with only occasional single cells adjacent to tumour cells. $\mathrm{B}$ and $\mathrm{T}$ lymphocytes were present in a ratio of approximately 1: 2, again with a predominance of CD4 cells being found.

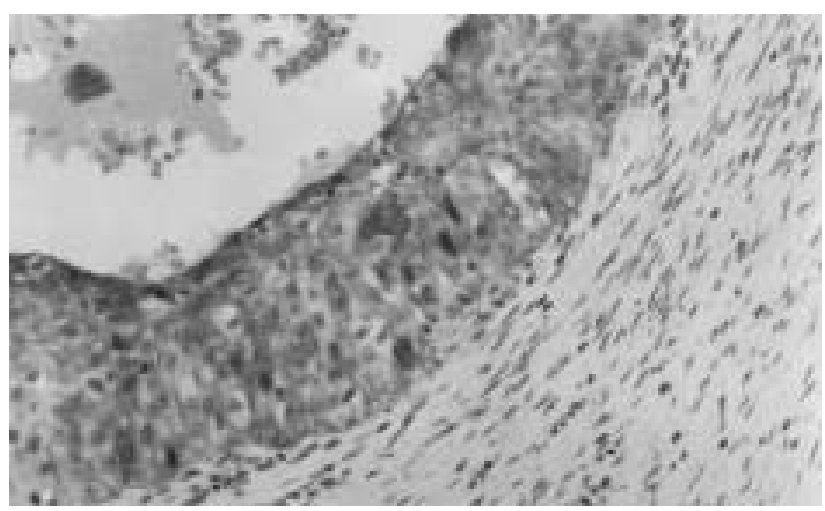

Fig. 2: HLA class II (DR) expression in comedo ductal carcinoma in situ (DCIS). Immunostaining using APAAP technique (red labeled cells). Original magnification X200.

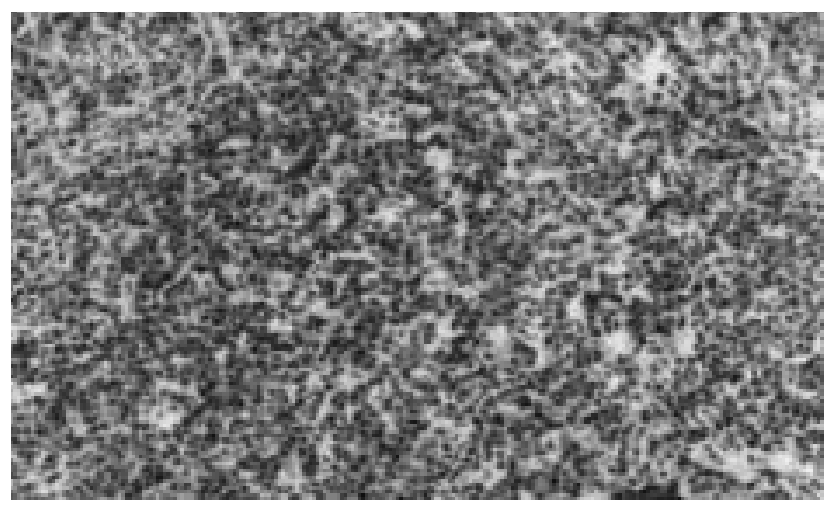

Fig. 4: Atypical ductal hyperplasia (ADH): Stromal T lymphocytes positive for the immunohistochemical stain directed against the CD4 antigen (stain, alkaline phosphatase antialkaline phosphatase; original magnification, X200). 


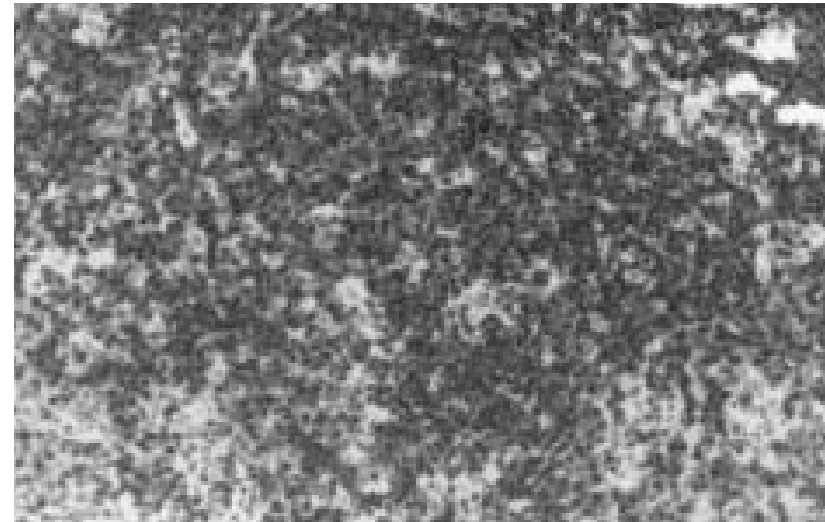

Fig. 5: Infiltrating ductal carcinoma (IDC-NOS): Stroma T lymphocytes positive for the immunohistochemical stain directed against the CD4 antigen (stain, alkaline phosphatase antialkaline phosphatase; original magnification, X200).

Tab. 2:

Average numbers of HLA class II (DR)+ mammary epithelial cells in ADH, DCIS, and IDC-NOS per high power field (range in brackets).

\begin{tabular}{|l|c|c|c|}
\hline & ADH & DCIS & IDC-NOS \\
\hline HLA II (DR) & 30.7 & 26.6 & 2.85 \\
antibody & $(4.7-142)$ & $(0.3-60)$ & $(0.3-11.1)$ \\
\hline
\end{tabular}

Average numbers of CD4+ mammary stromal cells in ADH, DCIS, and IDC-NOS per HPF (range in brackets)

\begin{tabular}{|l|c|c|c|}
\hline & ADH & DCIS & IDC-NOS \\
\hline CD4 & 3.3 & 22.9 & 41 \\
antibody & $(0.2-9.7)$ & $(0.5-74)$ & $(4.7-89)$ \\
\hline
\end{tabular}

Quantification - Stromal lymphocytes identified by each monoclonal antibody were present in far greater numbers in DCIS and IDC-NOS as compared with ADH $(\mathrm{p}<0.001)$.

Epithelial Staining - It was observed that all monoclonal antibodies directed against lymphocytes also reacted with both non-malignant and malignant epithelium in a substantial number of cases. However, intensity of staining was generally weak and did not interfere with the recognition and quantification of infiltration of lymphocytes.

Interrelationship of epithelial HLA class II (DR) antigens and stromal infiltrate - DCIS and IDC-NOS with greater HLA class II (DR) expression had more intense stromal infiltration by total $\mathrm{T}$ lymphocytes, this being reflected by the CD4 subsets in both instances. Carcinomas with loss of HLA II (DR) expression had slightly fewer CD4 positive cells.

Table 2 shows the average numbers of HLA class II (DR)+ and CD4+ cells in atypical ductal hyperplasia, ductal carcinoma in situ, and infiltrating ductal carcinoma of the breast, respectively.

\section{Discussion}

In the present study, we clearly demonstrated a loss of HLA class II (DR) expression from atypical ductal hyperplasia towards invasive carcinoma.

It is well known that HLA class II antigens are usually expressed on such immune cells as macrophages, B cells and activated $T$ cells and that they are also involved in antigen presentation as well as in the regulation of the helper $T$ cell function. A number of studies have also revealed the expression of class II antigens by both various non-immune normal and malignant cells $(1,2,4,14,33,38,48,45)$, although the biological significance of the class II expression of such cells remains unclear.

The presence of epithelial hyperplasia is the most important risk factor for subsequent malignancy. Fibrocystic changes increase progressively in women through the childbearing years, reach a peak during the perimenopausal period, and regress after menopause (2). Although the various components of fibrocystic change are usually found together, it has become increasingly clear that this is a heterogeneous group of lesions that should be diagnosed separately (17). The extent and type of epithelial proliferation found in these biopsy specimens is a major predictor for the subsequent development of mammary carcinoma, whereas the other components are of little significance in that regard (13). In addition, epidemiological similarities to breast cancer apply most consistently to the subset of patients with significant epithelial atypia (36).

Atypical ductal hyperplasia (ADH) accounts for less than $3 \%$ of all breast lesions found at biopsy. This is probably an underestimate of its incidence because it lacks distinctive features that would bring it to medical attention. It is most commom in postmenopausal women. Several studies have shown that women with diagnosed ADH have a relative risk of 4.0 to 5.0 for the subsequent development of invasive carcinoma $(12,13,28)$.

A common criticism is that the reproducibility of diagnosing $\mathrm{ADH}$ is low, and this has been attributed to a lack of consensus regarding its histologic criteria (39). Recent studies have taken an empirical approach to defining $\mathrm{ADH}$ that is based on evaluating the association between worrisome proliferative lesions (irrespective of traditional concepts of dysplasia) and the clinical outcome (12,13). Reproducible diagnostic criteria have been defined (42), and these have been used repeatedly to validate $\mathrm{ADH}$ as a strong risk factor for breast cancer $(12,13)$.

There is a controversy regarding whether ADH is merely a risk factor, or also a precursor lesion for breast cancer. Some authors have suggested that $\mathrm{ADH}$ is a small noninvasive carcinoma, basing their speculation on the close histologic resemblance between them. However, noninvasive carcinoma has a twofold to threefold stronger association than $\mathrm{ADH}$ does with the subsequent development of invasive carcinoma, and this risk is ipsilateral for noninvasive carcinoma (35), but bilateral for $\operatorname{ADH}(12,28)$. 
Noninvasive carcinoma is also often seen in continuity with concurrent invasive disease, whereas ADH is not. These data and observations indicate that noninvasive carcinoma may be both a risk factor and a non obligatory precursor for invasive carcinoma, though $\mathrm{ADH}$ may be a marker of increased risk (8). However, this issue is far from settled, and is likely to remain so until more is known about the biology of breast cancer evolution.

On the other hand, in view of immunological aspects, the class II expression of tumor cells has been reported to correlate with the local infiltration of lymphocytes $(10,23)$. There have been reported studies examining antigen presentation by epithelial cells from the human breast $(7,15)$.

In the present study, expression of HLA class II (DR) by epithelial neoplastic cells was possibly mediated by stromal T helper lymphocytes as lymphoid cell infiltrates were observed to all biopsy specimens containing HLA class II (DR) positive neoplastic cells. The increased aberrant expression of HLA II in tumor cells has been viewed as an important feature to escape tumor recognition by immune cells, and correlates with high grade malignancy and enhanced metastatic potential. In our series of breast lesions, there was a decreased expression of HLA II as the neoplastic process progressed to malignancy and a subsequent increased immune response, providing new insights for a better understanding of the tumorhost relationships in this form of neoplasia.

MHC tumor expression before, during, or after immunotherapy may be a necessary step in tumor response to treatment (15). Breast cancer has low activity of tumor infiltrating lymphocytes, and immunotherapy has not shown any advantage. Studies have shown that malignancies that respond to interleukin (IL)-2 treatment express HLA-DR before treatment, whereas non-responding tumors do not, either before or after treatment. Likewise, malignant melanomas transfected with MHC-I genes showed better responses to IL-2 treatment, and MHC-I induction in a highly tumorigenic adenovirus- 2 transformed cell line resulted in the complete loss of oncogenicity. Therefore, we suggest that immunotherapy with a cocktail of cytokines known to induce MHC-II expression may induce breast tumor cells to express MHC-II antigens. This could increase their immunogenicity and susceptibility to cytotoxic and helper $\mathrm{T}$ cells, respectively, and activate lymphocytic infiltrates to proliferate in situ.

\section{Acknowledgment}

We thank Irene Apostolou for her excellent technical assistance in the immunohistochemical studies.

\section{References}

1. Bartek J, Petrek M, Vogtesek B et al. HLA-DR antigens on differentiating human mammary gland epithelium and breast tumors. Br J Cancer 1987;56:727-33.

2. Bartow SA, Pathak DR, Black WC, Key CR, Teaf SR. Preavalence of benign, atypical and malignant breast lesions in populations at different risk for breast cancer: a forensic autopsy study. Cancer 1987;60:2751-60.
3. Bernard D, Maurizis JC, Ruse F et al. Presence of HLA-D/DR antigens on the membrane of breast tumor cells. Clin Exp Immunol 1984;56:215-21.

4. Brunner CA, Gokel JM, Riethmller G et al. Expression of HLA-D subloci DR and DQ by breast carcinomas is correlated with distinct parameters of favorable prognosis. Eur J Cancer $1991 ; 27: 411-6$.

5. Concha A, Esteban F, Cabrera $\mathrm{T}$ et al. Tumor aggressiveness and $\mathrm{MHC}$ class I and II antigens in laryngeal and breast cancer. Semin Cancer Biol 1991;2:47-54.

6. Concha A, Cabrera T, Ruiz-Cabello P et al. Can the HLA phenotype be used as a prognostic factor in breast carcinomas? Int J Cancer 1991;6(suppl):146-54.

7. Concha A, Ruiz-Cabello F, Cabrera T, Nogales F, Collado A, Garrido F. Different patterns of HLA-DR antigen expression in normal epithelium, hyperplastic and neoplastic malignant lesions of the breast. Eur J Immunogenet 1995;22(4):299-310.

8. Connolly JL, Schnitt SJ. Benign breast disease: resolved and unresolved issues. Cancer 1993:71:1187-9.

9. Cordell JL, Falini B, Erber WN et al. Immunoenzymatic labeling of monoclonal antibodies using immune complexes of alkaline phosphatase and monoclonal anti-alkaline phosphatase (APAAP complexes). J Histochem Cytochem 1984; 32:219-29.

10. Dammrich J, Buchwald J, Papadopoulos T, Muller-Hermelink HK. Special subtypes of pulmonary adenocarcinomas indicated by different tumor cell HLA-expression and stromal infiltrates. Virchows Arch B Cell Pathol 1991;61:9-18.

11. Doyle A, Martin WJ, Funa K. Markedly decreased expression of class I histocompatibility antigens protein, and mRNA in human small cell lung cancer. J Exp Med 1985; 161:1135-51

12. Dupont WD, Page DL. Risk factors for breast cancer in women with proliferative breast disease. N Engl J Med 1985;312:146-51.

13. Dupont WD, Parl FF, Hartmann WH et al. Breast cancer risk associated with proliferative breast disease and atypical hyperplasia. Cancer 1993;71:1258-68.

14. Esteban F, Concha A, Huelin C et al. Histocompatibility antigens in primary and metastatic squamous cell carcinoma of the larynx. Int J Cancer 1989;43:436-42.

15. Feinmesser M, Sulkes A, Morgenstern S, Sulkes J, Stern S, Okon E. HLA-DR and beta 2 microglobulin expression in medullary and atypical medullary carcinoma of the breast: histopathologically similar but biologically distinct entities. J Clin Pathol 2000;53(4):286-91.

16. Ghosh AK, Moore M, Street AJ et al. Expression of HLA-D sub-region products on human colorectal carcinomas. Int J Cancer 1986;38:459-64.

17. Godfrey SE: Is fibrocystic disease of the breast precancerous? Arch Pathol Lab Med 1986;110(11):991.

18. Goodenow RS, Vogel JM, Linsk RL et al. Histocompatibility antigens on murine tumors. Science 1985;230:777-83.

19. Gourley C, Thornton C, Massie C et al. Is there a relationship between HLA type and prognostic factors in breast cancer? Anticancer Research 2003;23:633-8.

20. Hämmerling G, Maschek U, Sturmhöfel K et al. Regulation and functional role of MHC expression on tumors. In: Melchers F, Albert ED, Von Boehmer AH et al, eds. Progress in immunology, vol 7. Berlin: Springer-Verlag, 1989:pp1071-8.

21. Holden CA, Sanderson AR, MucDonald DM. Absence of human leukocyte antigen molecules in skin tumors and some cutaneous appendages: evidence using monoclonal antibodies. J Am Acad Dermatol 1983;9:567-71.

22. Hurlimann J, Saraga P. Mononuclear cells infiltrating human mammary carcinomas: Immunohistochemical analysis with monoclonal antibodies. Int $\mathrm{J}$ Cancer 1985:35:753-62.

23. Kamma H, Yazawa T, Ogata T, Horiguchi H, Iijima T. Expression of MHC class II antigens in human lung cancer cells. Virchows Arch B Cell Pathol 1991;60: $407-12$.

24. Kaufman JF, Auffray C, Korman AJ et al. The class II molecules of the human and murine major histocompatibility complex. Cell 1984;36:1-13.

25. Koretz K, Moldenhauer G, Majdic O et al. Correlation of HLA-D/Ii antigen expression in breast carcinoma with local lymphohistiocytic infiltration reveals considerable dysregulation in a subset of tumors. In J Cancer 1989:44:816-23.

26. Lampson LA, Fisher CA, Whelan JP. Striking paucity of HLA-A, B, C and beta2-microglobulin on human neuroblastoma cell lines. J Immunol 1983;130: 2471-8.

27. Lazaro B, Anderson A, Kajdacsy-Balla A, Hessner M. Antigenic characterisation of medullary carcinoma of the breast: HLA-DR expression in lymph node positive cases. Appl Immunohistochem Molecul Morphol 2001;9:234-41.

28. London SJ, Connolly JL, Schnitt SJ, Colditz GA. A prospective study of benign breast disease and the risk of breast cancer. JAMA 1992;267:941-4

29. Lucin K, Iternicka Z, Jonjic N. Prognostic significance of T-cell infiltrates, expression of 2-microglobulin and HLA-DR antigens in breast carcinoma. Pathol Res Pract 1984;190:1134-40.

30. Müller C, Ziegler A, Müller C et al. Divergent expression of HLA-DC/MB, -DR and $-\mathrm{SB}$ region products on normal and pathological tissues as detected by monoclonal antibodies. Immunbiology 1985;169:228-49.

31. Moller P, Mattfeldt T, Gross C et al. Expression of HLA-A, -B, -C, -DR, -DQ, and of HLA-D-associated invariant chain (Ii) in non-neoplastic mammary epithelium, fibroadenoma, adenoma and carcinoma of the breast. Am J Pathol 1989;135: $73-83$. 
32. Natali PG, Giacomini P, Bigotti A et al. Heterogeneity in the expression of HLA and tumor-associated antigens by surgically removed and cultured breast carcinoma cells. Cancer Res 1983;43:660-8

33. Natali P, Bigotti A, Cavalieri R et al. Gene products of the HLA-D region in normal and malignant tissues of nonlymphoid origin. Hum Immuno $1986 ; 15: 220-33$

34. Norton AJ, Jordan S, Yeomans P. Brief, high-temperature heat denaturation (pressure cooking): a simple and effective method of antigen retrieval for routinely processed tissues. J Pathol 1994;173:371-9.

35. Page DL, Dupont WD, Rogers LW, Landenberger M: Intraductal carcinoma of the breast: follow up after biopsy only. Cancer 1982;49:751-8.

36. Parazzini F, La Vecchia C, Franceschi S et al. Risk factors for pathologically confirmed benign breast disease. Am J Epidemiol 1984;120:115-22.

37. Perez M, Cabrera T, Lopez Nevot MA et al. Heterogeneity of the expression of class I and II HLA antigens in human breast carcinoma. J Immunogenet 1986;13:247-53.

38. Redondo M, Concha A, Oldiviela R et al. Expression of HLA class I and II antigens in bronchogenic carcinomas: its relationship to cellular DNA content and clinical-pathological parameters. Cancer Res 1991;51:4948-54.

39. Rosai J. Borderline epithelial lesions of the breast. Am J Surg Pathol 1991; $15: 209-21$.

40. Rosenberg SA. Progress in human tumour immunology and immunotherapy. Nature 2001;411:380-4

41. Rubin JT, Elwood LJ, Rusenberg SA et al. Immunohistochemical correlates of response to recombinant interleukin-2-based immunotherapy in humans. Cancer Res 1989:49:7086-92.

42. Schnitt SJ, Connolly JL, Tavassoli FA et al. Interobserver reproducibility in th diagnosis of ductal proliferative breast lesions using standardized criteria. Am J Surg Pathol 1992;16:1133-43.

43. Schrier PI, Bernards R, Vaessen RT et al. Expression of class I major histocompatibility antigens switched off by highly oncogenic adenovirus 12 in transformed rat cells. Nature 1983;305:771-5
44. Tsuda $\mathrm{H}$, Hirohashi $\mathrm{S}$, Higuchi $\mathrm{K}$ et al. Beta-2-microglobulin expression in relation to amplification of oncogenes and prognosis in breast carcinoma. Histopathology 1990;16:600-2.

45. van Duinen SG, Ruiter DJ, Broecker EB et al. Level of HLA antigens in locoregional metastases and clinical course of the disease in patients with melanoma. Cancer Res 1988;48:1019-25.

46. Vogel J, Tanaka K, Hoekzema GS et al. Experimental strategies for modification of histocompatibility antigen in tumor cell. Cancer Metastasis Rev 1987;6: $677-93$

47. Weiss MA, Michael JG, Pesce AJ et al. Heterogeneity of 2-microglobulin in human breast carcinoma. Lab Invest 1981;45:46-57.

48. Wintzer HO, Benzing M, von Kleist S. Lacking prognostic significance of 2 microglobulin, MHC class I and class II antigen expression in breast carcinomas. Br J Cancer 1990;62:289-95.

49. Zuk JA, Walker RA. Immunohistochemical analysis of HLA antigens and mononuclear infiltrates of benign and malignant breast. J Pathol 1987:152:275-85.

50. Zuk JA, Walker RA. HLA class II sublocus expression in benign and malignan breast epithelium. J Pathol 1988;155:301-9.

Submitted May 2004.

Accepted July 2004.

Nikolas Papadopoulos,

Assoc. Prof. in Histology-Embryology,

Democritus University of Thrace,

Dragana, 68100 Alexandroupolis,

Greece.

e-mail: npapad@med.duth.gr 